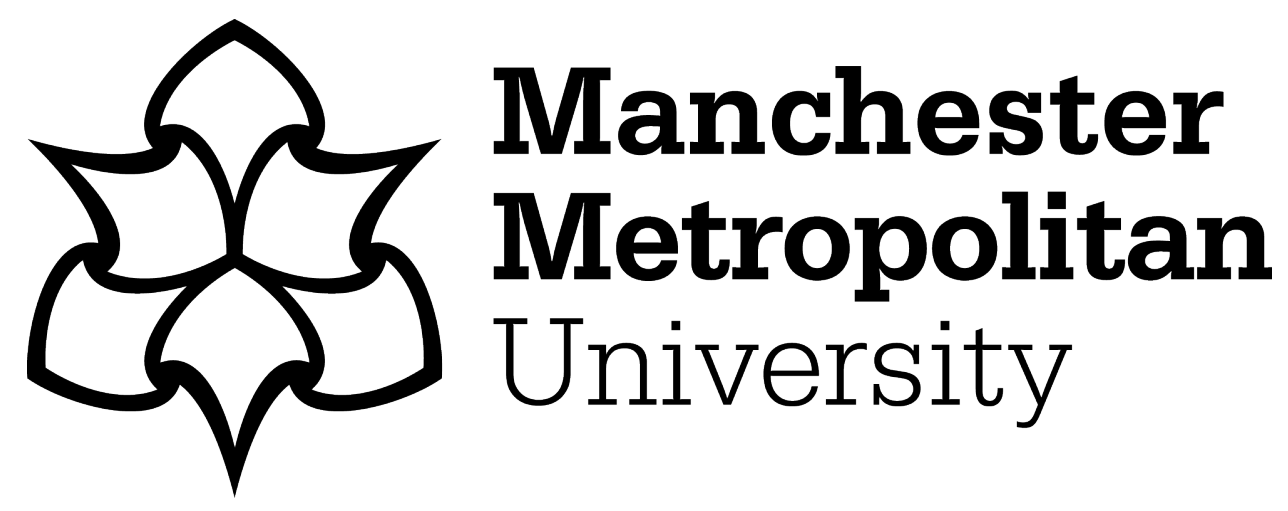

Grant, RA, Delaunay, MG and Haidarliu, S (2017) Mystacial whisker layout and musculature in the guinea pig (Cavia porcellus): a social, diurnal mammal. The Anatomical Record, 300 (3). pp. 527-536. ISSN 1932-8486

Downloaded from: https://e-space.mmu.ac.uk/617394/

Version: Accepted Version

Publisher: Wiley

DOI: https://doi.org/10.1002/ar.23504

Please cite the published version 


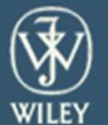

The Anatomical Record

\section{Mystacial whisker layout and musculature in the guinea pig (Cavia porcellus): a social, diurnal mammal.}

\begin{tabular}{|r|l|}
\hline Journal: & Anatomical Record \\
\hline Manuscript ID & AR-16-0159.R1 \\
\hline Wiley - Manuscript type: & Full Length Article \\
\hline Complete List of Authors: & $\begin{array}{l}\text { Grant, Robyn; Manchester Metropolitan University } \\
\text { Delaunay, Mariane; Manchester Metropolitan University } \\
\text { Haidarliu, Sebastian; The Weizmann Institute of Science, Neurobioligy }\end{array}$ \\
\hline Keywords: & vibrissae, active sensing, touch, rodent, facial muscles \\
\hline
\end{tabular}

\section{SCHOLARONE ${ }^{\mathrm{m}}$}

Manuscripts 
1 Mystacial whisker layout and musculature in the guinea pig (Cavia porcellus): a social, 2 diurnal mammal.

3 Running title: Guinea pig whisker layout and musculature

4 Robyn A. Grant ${ }^{1 *}$, Mariane G. Delaunay ${ }^{1}$ and Sebastian Haidarliu ${ }^{2}$

5 1. Conservation, Evolution and Behaviour Research Group, Manchester Metropolitan

$8 \quad *$ Contacting Author:

$9 \quad$ Email:robyn.grant@mmu.ac.uk, Tel: +44 (0)161 2476210, Fax: 44 (0)161 2476840

10

24 Keywords: vibrissae, active sensing, touch, rodent, facial muscles, evolution 


\section{Abstract}

27 All mammals (apart from apes and humans) have whiskers that make use of a similar muscle

28 architecture. Whisker specialists, such as rats and mice, tend to be nocturnal and arboreal,

29 relying on their whisker sense of touch to guide exploration around tree canopies at night. As

30 such, nocturnal arboreal rodents have many whiskers that are organised into a grid-like

31 pattern, and moved using a complex array of muscles. Indeed, most arboreal, nocturnal

32 mammals tend to have specialised whiskers, that are longer and arranged in a dense, regular

33 grid, compared to terrestrial, diurnal mammals. The guinea pig diverged early from murid

34 rodents (around 75 million years ago), and are ground-dwelling, diurnal animals. It would be

35 predicted that, as a terrestrial mammal, they may have less whiskers and a reduced muscle

36 architecture compared to arboreal, nocturnal rodents. We examined the mystacial whisker

37 layout, musculature and movement capacity of Guinea pig (Cavia porcellus) whiskers and

38 found that they did indeed have a disorganized whisker layout, with a fortification around the

39 eye area. In addition, there was a reduction in musculature, especially in the intrinsic muscles.

40 Despite guinea pigs not cyclically moving their whiskers, the mystacial musculature was still

41 very similar to that of murid rodents. We suggest that the conserved presence of whisker

42 layout and musculature, even in visual mammals such as primates and guinea pigs, may

43 indicate that whiskers still play an important role in these animals, including protecting the

44 eyes and being involved in tactile social behaviours. 


\section{Introduction}

47 All mammals (apart from apes and humans) have facial whiskers (vibrissae) (Ahl, 1976).

48 Whisker specialists, such as mice, rats and hamsters are able to move their whiskers to

49 perform large, quick, cyclic sweeps (termed whisking), which is amongst the fastest

50 movements that mammals can make (occurring at around $25 \mathrm{~Hz}$ in mice) (Welker, 1964;

51 Wineski, 1983; Jin et al., 2004). The fast and precise positioning of the whiskers are enabled

52 by a specialist whisker musculature, a complicated architecture of intrinsic and extrinsic muscles (Dörfl, 1982), which are represented mainly by fast muscle fibres (Jin et al., 2004). Perhaps the most well-studied muscle group is that of the intrinsic muscles, represented by sling-like muscles that link around the base of each whisker follicle, causing the whiskers to protract forward (Dörfl, 1982). The layout of the intrinsic muscles has been found to be largely preserved from marsupials (Grant et al., 2013) to rodents (Dörfl, 1982), to nocturnal arboreal primates (Muchlinski et al., 2013). That intrinsic whisker muscles are preserved between marsupials and rodents, even though their last common ancestor occurred around 160 million years ago (Luo et al., 2011), suggests that the common ancestor of extant mammals may well have had moveable whiskers involved in active touch sensing. While intrinsic muscles are largely preserved, the extrinsic muscles of the mystacial pad can largely vary between species (Yohro, 1977). In the marsupial Monodelphis domestica (Grant et al., 2013), for example, some of the extrinsic muscles are so reduced that vibrissal control is limited, and whisker spread and velocity cannot be controlled during object exploration.

The number, layout and musculature of the mystacial vibrissae are all closely linked to the function and movement abilities of the whiskers. Small, social, nocturnal and arboreal mammals have been found to have longer vibrissae with a more densely packed vibrissal field than that of ground-dwelling and burrowing mammals (Pocock, 1914; Lyne, 1959; Ahl, 1986; Muchlinski et al., 2013). Exceptions to this include semi-aquatic (i.e. Australian water rat (Dehnhardt et al., 1999)) and aquatic mammals (such as pinnipeds and sirenians (Dehnhardt, 2002)), that have long and densely-arranged whiskers, despite them being large, diurnal animals; indeed the California sea lion has the longest whiskers of all mammals. In these animals, the whiskers are a likely adaptation for an aquatic lifestyle, and are used for navigation and prey capture in a dark, underwater environment (Grant \& Arkley, 2016).

77 Arboreal, nocturnal rodents actively position their whiskers for use in a variety of functions, including navigation, locomotion, exploration, hunting and social touch (Grant \& Arkley, 
79 2016). Extensive studies in arboreal, nocturnal mice and rats have revealed that they possess

80 three groups of whisking muscles (protractors, retractors, and vertical vibrissae deflectors)

81 leading to a range of whisker movements and vast control abilities (Haidarliu et al., 2010).

82 However, diurnal mammals, such as some primates, lack organized vibrissae, have very thin

83 whiskers and a reduced whisker follicle without intrinsic muscles (Muchlinski et al., 2013).

85 Guinea pigs, and other Hystricomorphs, diverged from the murid rodents before the

86 artiodactyls and primates, and are often thought of as a separate order from rodentia (Graur et

87 al., 1991). They represent an early divergence in eutherian evolution, and as such often have

88 rather anomalous characteristics compared to other mammals, such as their facial bone

89 structure (Muchlinski, 2008) and body muscles (Potter et al., 1957). The guinea pig, and

90 other Histricomorphs, have a unique facial anatomy, in that the media masseter (mastication)

91 muscle passes through the infraorbital foramen (IOF) of the skull, which makes it particularly

92 large, compared to the IOF of other rodents (Muchlinski, 2008). While some aspects of the

93 maxilliary facial musculature has been described in guinea pigs (Muchlinski 2008), that of

94 the mystacial pad has yet to be considered, despite guinea pigs being able to generate fast and

95 large amplitude whisker movements; however, these movements do not tend to be cyclic and

96 usually occur in isolation (Jin et al., 2004). Due to their early divergence, we might expect the

97 guinea pig to have a whisker layout and musculature more similar to the marsupial, than the

98 rodent tactile specialists that evolved later, such as mice and rats. Moreover, we might expect

99 the guinea pig whisker system to be even further reduced and disorganized, due to them being

100 diurnal, ground-living mammals.

101

102 The aim of this study is to describe the muscle architecture of the mystacial pad in the guinea

103 pig anatomically, by cutting the mystacial pads of the guinea pig in the tangential plane and

104 staining consecutive slices for cytochrome c oxidase activity and Masson's Trichrome.

105 Because of the differences in proposed up-to-date schemes of whisker layouts in the guinea

106 pig mystacial pad (Sikich et al., 1986; Haidarliu and Ahissar, 1997), we also re-examine here

107 the layout of the mystacial pad in situ. All results will be compared in detail with those of rat,

108 and also to other animals such as opossums and shrews. We will go on to consider the impact

109 of diurnality on the whisker pad muscles and layout.

110

111

4

John Wiley \& Sons, Inc. 


\section{Materials and Methods}

\section{Pad removal}

114 Eight female adult Dunkin-Hartley guinea pigs were used in the anatomy section of this 115 study, each weighing between $350-400 \mathrm{~g}$. The guinea pigs were euthanized via an overdose

116 of anaesthetic. The mystacial pads were removed by cutting down the skin in the sagittal

117 plane and around each pad (around $2 \mathrm{~mm}$ on either side of the pad). They were placed into a

118 solution of fixative (4\% paraformaldehyde in $0.1 \mathrm{M}$ phosphate buffer) and left for one hour.

119 They were then straightened by placing them into histology cassettes (Medex Supply,

120 Monsey, NY, USA) packed with high-density foam. Twelve of the pads were then placed

121 into fixative solution enriched with sucrose up to $30 \%$ for twenty four hours, and then

122 sectioned on the freezing microtome for staining for cytochrome oxidase activity. The

123 remaining four pads were kept flattened for two weeks, then subjected to dehydration and

124 clearing, and mounted in paraffin wax to slice and stain with Mason's Trichrome.

\section{Staining for cytochrome oxidase activity}

126 After fixing, each of the pads was sectioned using a freezing microtome (Leica CM 1800)

127 into $60 \mu \mathrm{m}$ thick slices in the tangential plane. All slices were stained for cytochrome oxidase

128 activity following the method developed by Wong-Riley (1979) and modified by Haidarliu et 129 al. (2010). Slices were floated in an oxygenated solution of $0.02 \%$ cytochrome c $(0.75 \mathrm{mg})$, 130 catalase solution $(40 \mu \mathrm{l})$, and $0.05 \%$ diaminobenzidine $(5 \mathrm{mg})$ in $0.1 \mathrm{M}$ phosphate buffer. The 131 slices were incubated at room temperature on a shaking platform until the stain developed 132 (approximately 1-3 hours), and a clear differentiation between non-reactive and highly 133 reactive tissue structures could be determined. Slices were then rinsed with $0.1 \mathrm{M}$ phosphate 134 buffer. Stained slices were mounted on microscope slides and left to air dry overnight. The 135 slices were then coverslipped with DPX.

136 The cross-sectional diameter of the intrinsic muscles was manually measured, perpendicular 137 to the follicle, on the C-row whisker follicles of one slide (Fig. 3B) using the image analysis 138 software Tracker (Brown 2015), and compared to an equivalent slice in rat.

139 Staining with Masson's Trichrome

140 Four of the pads were placed into empty histology cassettes and transferred to a tissue 141 processor (Shandon Citadel 2000), where tissues were dehydrated through a series of graded 
142 IMS baths $(70 \%, 80 \%, 90 \%, 100 \%)$, and then immersed in xylene and paraffin wax. This

143 process took around 20 hours. The samples were then mounted in a block of paraffin wax and

144 sliced on an automatic rotary microtome (Thermos Scientific microtome HM355S) into 10

$145 \mu \mathrm{m}$ thick slices that were floated in a $35-37^{\circ} \mathrm{C}$ bath. Two mystacial pads were sliced

146 tangentially, and two were sliced horizontally to visualise the C-row follicles. Slices were

147 mounted onto slides and left to dry at $38^{\circ} \mathrm{C}$ overnight.

148 Slides were put in a fixative solution (4\% paraformaldehyde in $0.1 \mathrm{M}$ phosphate buffer) for 1

149 hour, and introduced to Bouin's Solution for 4 hours. They were then cleared with xylene, 150 rehydrated with ethyl alcohol $(100 \%, 90 \%, 80 \%, 70 \%)$ and moved through a sequence of

151 solutions for the Masson's Trichrome staining (Biebrich Scarlet Acid, Phosphotungstic and

152 Phosphomolybdic Acids, Aniline Blue and Acidified Water), with multiple washes of

153 distilled water in-between each stage. The slices were then dehydrated with ethyl alcohol

$154(70 \%, 80 \%, 90 \%, 100 \%)$ and xylene, towel dried and cover-slipped using DPX. All slices

155 were visualised using a Zeiss Stereo Lumar V12 light microscope. Figures were captured

156 using Zeiss Axiovision, version 4.8. Occasional adjustments to exposure and white balance

157 were made.

158 Behavioural Filming

159 Nine adult female guinea pigs, of mixed strains, were used for filming. They were placed

160 individually into a transparent, Perspex, rectangular arena $(20 \times 50$ x $15 \mathrm{~cm})$ (Fig. 1A), which

161 was lit from below by an infrared light box (PHLOX LEDIR-BL-200/200-SLLUB-Q-1R-

162 24V). Each guinea pig was filmed from above using a digital high-speed video camera

163 (Phantom Miro ex2) recording at 500 frames per second with a shutter-velocity of $1 \mathrm{~ms}$ and a

164 resolution of 640x480 pixels. Multiple 1.5-s video clips were collected opportunistically (by

165 manual trigger) when the animal moved in the cameras field of view. Approximately 12 clips

166 were collected from each animal. Two-three clips from each guinea pig were selected based

167 on to the following selection criteria: i) the guinea pig was clearly in frame; ii) both sides of

168 the face were visible; iii) the head was level with the floor (no extreme pitch or yaw); and iv)

169 the whiskers were not in contact with a vertical wall. Twenty two clips in total were tracked

170 using the BIOTACT Whisker Tracking Tool (Perkon et al., 2011). The tracker semi-

171 automatically finds the orientation and position of the snout, and the angular position (relative

172 to the midline of the head) of each identified whisker. Tracking was validated by manually

173 inspecting the tracking annotations overlaid on to the video frames (Fig. 1B, inset). 
174 The movement of the entire whisker field was determined from the unsmoothed mean of all 175 the tracked whisker angular positions for each side frame by frame (Grant et al., 2013), which 176 can be seen in Fig. 1B and is termed naïve mean angle (nma). The offset was calculated as the 177 mean nma, and an average was taken between the two whisker sides. To estimate the 178 amplitude, the offset was removed from the whisking angle time series and the root mean 179 square value was computed to give the root-mean-square (RMS) whisking amplitude and was 180 estimated by multiplying the RMS whisking amplitude by $2 \sqrt{ } 2$ (Chatfield, 2003). Whisk 181 frequency was not calculated as the $n m a$ did not often contain clear whisks, rather they were 182 more asymmetric movements that oriented the whisker field.

183 All work in this study conformed to UK Home Office Regulations and was approved by local 184 ethics committees.

Results

Mystacial Pad Layout

188 The layout of the guinea pig whiskers indicates that there are five rather irregular rows of 189 whiskers within the mystacial pad (Fig. 2). Dorsal to these five rows of mystacial vibrissae 190 and to the nostril, a row of five-to-six arcwise arranged nasal vibrissae passes in the rostro191 dorsal direction. The most dorsal row of the mystacial vibrissae, row A, is made of only two 192 whiskers. Row B comprises of usually three, but sometimes four vibrissae. Rows A and B are 193 caudally straddled by a straddler $(\alpha)$. Each of the rows C, D, and E contains usually five 194 vibrissae. Rows D and E are positioned more rostral, such that vibrissae D1 and E1 align with 195 vibrissae $\mathrm{A} 2, \mathrm{~B} 2$ and $\mathrm{C} 2$. The misalignment of the rows $\mathrm{D}$ and $\mathrm{E}$ with rows $\mathrm{A}-\mathrm{C}$ reveals the 196 guinea pig to have a more irregular whisker pad than other rodents, for example rat (compare 197 with Fig. 3A).

198 The straddler whiskers of the guinea pig also have a complex arrangement. Straddlers $\gamma$ and $199 \delta$ sit ventro-caudal to the rest of the whisker pad, and it is not clear from just looking at the 200 layout in Fig. 2, which whisker rows they are associated with. Straddler $\alpha$ sits caudal to rows 201 A and B. Straddler $\beta$ straddles rows B and C. Muscle fibers from the ventral side of the 202 follicle $\mathrm{C} 1$, the dorsal side of the follicle D1 and straddler $\delta$ reach the follicle of the straddler $203 \gamma$ which is positioned more caudal to them (Fig. 3B). This is a rather irregular straddler 
204 layout, compared to the rat (Fig. 3A) where straddler whiskers straddle consecutive rows A-

205 B, B-C, C-D, and D-E.

206 Intrinsic Muscles

207 The guinea pig whisker pad contains sling-like intrinsic muscles (Figs. 3B-D) that form a

208 sling around the rostral areas of each follicle and attach to the caudal follicle in the same row.

209 These muscles are striated and made up of red, pink and white striated muscle fibers (Fig.

210 3D). The intrinsic muscles look much reduced, are much thinner, and are not as striking as

211 those seen in the rat (compare Figs. 3A and 3B). Indeed, the cross-sectional diameter of the

212 intrinsic muscles (measured at the point of the arrows on Figs. 3A and 3B) show that the C

213 row intrinsic muscle diameter is $0.080 \pm 0.01 \mathrm{~mm}$ in rat and $0.061 \pm 0.004 \mathrm{~mm}$ in guinea pig,

214 despite the guinea pig being slightly bigger overall.

215 In addition, the intrinsic muscles are, on the whole, more irregular in the guinea pig. In the

216 rat, the intrinsic muscles connect each consecutive follicle within the same row, forming a

217 regular, chain-like architecture (Figure 3A). However, in the guinea pig, oblique intrinsic

218 muscles pass both between and within vibrissal rows. Figure $3 \mathrm{C}$ shows an oblique intrinsic

219 muscle passing between follicles in different rows, from the ventral part of the B1 follicle

220 attaching to the dorsal part of the $\mathrm{C} 1$ follicle. Figure $3 \mathrm{E}$ shows an oblique intrinsic muscle

221 passing within vibrissal rows, from the ventral part of A2 crossing to the dorsal part of A1.

222 These oblique intrinsic muscles are not observed in rat.

223 Whisker Follicles

224 The intrinsic muscles can also be observed in Fig. 4, which shows a slice containing the C-

225 row of whiskers. The muscles (in red) can be clearly seen linking the bottom of a more rostral

226 follicle to the distal end of a more caudal follicle (C4-C3, C3-C2, C2-C1). In addition, the

227 whisker C2 (the second whisker follicle from the right) contains a clear follicle sinus and

228 ringwulst. The sinus can also clearly be seen in the follicles in Fig. 3B and C.

229 Extrinsic Muscles

230 The superficial extrinsic muscles, M. nasolabialis and M. maxillolabialis, are both present.

231 They insert into the caudal areas of the mystacial pad, and merge rostrally between the rows

232 of vibrissae (Fig. 5A and B). The bundles of the Mm. maxillolabialis and nasolabialis fan

233 rostrally, each forming a thin layer, so that they can usually be seen clearly in different slices 
234 (Fig. 5A and B). Another superficial extrinsic muscle that participates in vertical vibrissa 235 spreading (Pars orbicularis oris of the M. buccinatorius) can also be seen in Fig. 2.

236 The deep vibrissa retracting muscles are part of the M. nasolabialis profundus. The Pars 237 interna profunda (PIP) occupies the most dorsal position in the rostral segment of the 238 mystacial pad. Its origin is represented by a number of tapered ends of muscle fibres that are 239 attached to the nasal cartilage. Muscle fibres fan and run toward rows A and B (Fig. 6A).

240 Guinea pigs possess a single Pars maxillaris that originates from a large area of the nasal 241 cartilage ventral to the PIP origin. It is not divided into two parts (superficialis et profunda), 242 as in many other rodents, and runs through and around rows $\mathrm{C}-\mathrm{E}$. The separation of the 243 deep vibrissa retracting muscles in to two groups, those targeting A and B rows and those 244 targeting rows C-E, may reflect compartmentalization of the guinea pig mystacial pad into 245 nasal and maxillary parts. The nasal and maxilliary compartments have been labelled on Figs. $2463 \mathrm{~A}$ and $\mathrm{B}$ in rat and guinea pig, and are also reflected in the higher density grouping of the 247 follicles in rows C-E, compared to A and B. The deep vibrissa retracting muscles submerge 248 near the proximal ends of the five vibrissal rows and insert into the deep fibrous mat that is represented, similar to rats, by thick collagenous bundles (Fig. 7A, C). The collagenous nature of these bundles was confirmed by their autofluorescence (Fig.7D).

251 The deep vibrissa protracting muscles can be seen in the mystacial pad slices as two groups 252 of densely arranged muscle bundles, that correspond to the Partes mediae superior et inferior 253 in other rodents (Fig. 7A). Their origins are not seen in tangential slices of the mystacial pad 254 because the nose of the guinea pig contains larger cartilages and well developed soft tissues, 255 compared with whisking rodents. Muscle bundles are cut transversally and contain three 256 types of muscle fibres (Fig. 8B and C), similar to those of the rat.

\section{Behaviour}

Behavioural data from the guinea pigs show that the whiskers are not moved in continuous cycles; rather, they remain stationary, until a large head rotation or forward movement occurs. Some cyclic movements (whisking) can be seen, but these only occur in short bouts (Fig. 1B, right whisker in blue). Most whisker movements are in isolation, asymmetric and do 263 not show clear whisking (Fig. 8C). The whiskers were positioned at mean offset values of $26498 \pm 12.5$ degrees, and moved with mean amplitudes of $44 \pm 25.9$ degrees (Fig. 8A and B). 


\section{Discussion}

267 The guinea pig is a ground-dwelling, diurnal mammal of the group Histricomorpha. As such,

268 we would expect to see a reduction in the number of whiskers and mystacial muscles,

269 compared to climbing, nocturnal rodents, such as rats and mice. We see here that the number

270 of whiskers are not only reduced in number, but also more irregularly distributed through the

271 pad. While the intrinsic and extrinsic mystacial musculature is largely conserved between

272 guinea pigs and rats, it is more irregular and somewhat reduced in the guinea pig. This has

273 implications for behaviour, with the guinea pig moving their whiskers in isolation and

274 asymmetrically, compared to the cyclic and almost continuous movements of whiskers

275 observed in rats and mice.

276 Whisker layout and follicles

277 The guinea pig mystacial pad has around 23 whiskers arranged in a grid-like layout. It

278 contains five rows of whiskers, which is the same as in rats and mice (Haidarliu et al., 2010).

279 However, each row in the guinea pig contains fewer whiskers, especially the most dorsal row

280 A, which only contains two whiskers (Fig. 2). Indeed, the guinea pig has much fewer

281 whiskers than hamsters (23 whiskers, Wineski, 1985; Haidarliu and Ahissar, 1997), rats (33

282 whiskers, Haidarliu et al., 2010), mice (33 whiskers, Dörfl, 1982), and even shrews (around

28340 whiskers Kulikov, 2011; Brecht et al. 2011) who have a much earlier evolutionary lineage

284 than guinea pigs. This reduction in whisker number in the guinea pig is, therefore, likely to be

285 associated with a diurnal, visual lifestyle, rather than simply being more primitive than rats

286 and mice.

287 As well as there being fewer whiskers in guinea pig, compared to rats and mice, the whiskers 288 are also more irregularly positioned (compare Fig. 3A and 3B). In rats, the straddler whiskers 289 are caudal to the main whisker rows, and sit between them in a regular fashion (Haidarliu et 290 al., 2010). In the guinea pig, straddlers $\alpha$ and $\beta$ sit fairly uniformly and are caudal and dorsal 291 to row B and C, respectively; however, $\gamma$ and $\delta$ do not align well with rows D and E (Fig. 2).

292 Whisker rows D and E are also displaced rostrally in the pad, such that D2 and E2 whisker

293 follicles are aligned with B3 and C3 (Fig. 2). The irregular organization of the whisker

294 follicles is also associated with a similar topographic disorganization of barrel structures in

295 the somatosensory cortex (Woolsey et al., 1975; Haidarliu et al., 1997). 
296 Individual whisker follicles in the guinea pig are large, and contain a clear follicle sinus and 297 ringwulst, similar to rats and opossums (Grant et al., 2013). This agrees with observations 298 from Rice et al. (1986), who found that guinea pig follicles were of a similar structure to 299 hamsters, mice, rats, gerbils, rabbits, guinea pigs and cats. Rice et al. (1986) measured the 300 degree of innervation in the guinea pig follicle, approximated by the number of axons in the 301 deep vibrissal nerve, and found it to be comparable to all these animals. However, 302 innervation of the inner conical body (the deep area of the follicle), in particular, was 303 decreased in the guinea pig and cat, compared to whisking animals such as the hamster, 304 mouse, rat and gerbil (Rice et al., 1986). This variation of innervation in the guinea pig 305 between the inner conical body and other areas of the follicle sinus complex (such as the 306 cavernous sinus and the ring sinus) caused the authors to conclude that innervation of the 307 guinea pig follicle was disorganized through the structure.

Musculature

309

The guinea pig mystacial pad contains intrinsic whisker muscles. This is relatively

310 unsurprising as intrinsic muscles have also been described in mice (Dörfl, 1982), hamsters

311 (Wineski, 1985), opossums (Grant et al., 2013), rats (Haidarliu et al., 2010), shrews (Yohro, 312 1977) and even nocturnal primates (Muchlinski et al., 2013), lending confidence to the view 313 that this is a primitive mammalian trait. The intrinsic muscles in guinea pigs are thinner than 314 those in rats (Fig. 3A and B) by around $0.02 \mathrm{~mm}$, despite guinea pigs being slightly larger 315 than the rats overall. In addition, the intrinsic muscles are also more irregular. For example, 316 two types of oblique intrinsic muscles occur in guinea pig; those that pass between follicles in 317 different rows, and those that connect follicles the same row (Fig. 3). Oblique intrinsic 318 muscles that connect follicles in neighboring rows (i.e. between B and C in Fig. 3C) are 319 relatively rare, and as yet have only been observed in the more ventral rows of the mystacial 320 pad in the big-clawed shrew ("straddling" muscles) (Yohro, 1977). The oblique intrinsic 321 muscles that connect follicles in the same row can be observed in the guinea pig in row A 322 (Fig. 3D). The position and attachment of these oblique intrinsic muscles in row A suggests 323 that they may cause a torsional rotation of the most dorsal whiskers, enabling the A row to 324 rotate during protraction. This type of oblique intrinsic muscle has only been observed before 325 in the opossum, Monodelphis domestica, which contains oblique intrinsic muscles in both the 326 A and B rows (Grant et al., 2013). In the opossum, the oblique intrinsic muscles were thought 327 to fortify the eye area (Grant et al., 2013), perhaps moving the whiskers in front of eye for 328 protection against collisions. The presence of these oblique intrinsic muscles in both the 
329 opossum and guinea pig may not, therefore, simply be representative of a disorganization of 330 the pad, but also lends support for the idea that whiskers could have a possible function in 331 protecting the eye area.

332 Superficial extrinsic muscles, that drive retraction movements of the vibrissae, are present in 333 the guinea pig (Fig. 5A and C), and have previously been described in hamsters (Wineski, 334 1985), mice (Dörfl, 1982; Klingener, 1964), rats (Haidarliu et al., 2010), jerboas (Klingener, 335 1964), opossums (Minkoff et al., 1979; Grant et al., 2013) and shrews (Yohro, 1977). There

336 is some variation between species, for example in the big-clawed shrew, the striated $M$.

337 nasolabialis superficialis is also associated with smooth muscle fibres just beneath the 338 corium (Yohro, 1977). In the guinea pig, these muscles look to be striated throughout, much 339 like in the rat and opossum (Grant et al., 2013; Haidarliu et al., 2010).

340 The guinea pig has deep vibrissa retracting muscles that are parts of the M. nasolabialis 341 profundus. They originate around the nose, run down most of the length of the mystacial pad 342 and pull the deep layers of the whisker pad forward, enabling the whiskers to retract back. In 343 mice and rats, these muscles belong to the bipennate type, indicating that their origins are 344 tendinous, and their attachment is limited by a small area of the nasal cartilage to which the 345 tendon is attached (Haidarliu et al., 2010, 2015). In the guinea pig, these muscles belong to a 346 divergent type; their origins are represented by multiple tapered ends of muscle fibres that 347 occupy a considerably larger surface of the nasal cartilage (Fig. 6). The fibres of such 348 muscles are long, and they fan in such a manner that their insertion sites are spread over a 349 large area reaching the deep fibrous mat of the mystacial pad. Similar fanning architecture of 350 the subunits of the M. nasolabialis profundus, and a single Pars maxillaris were also observed 351 in hamsters (Wineski, 1985).

352 Aspects of the deep retracting muscles have previously been described in mice (Dörfl, 1982;

353 Haidarliu et al., 2015; Klingener, 1964; Rinker, 1954), hamsters (Wineski, 1985), opossums

354 (Grant et al., 2013) and rats (Haidarliu et al., 2010; Rinker, 1954). In the opossum,

355 Monodelphis domestica, these muscles are greatly reduced, so much so the animal cannot

356 control retraction movements during contact (Grant et al., 2013). That these muscles are

357 almost absent in the opossum, but present in the guinea pig indicates that the deep retracting 358 muscles might have become more established in a common ancestor of guinea pigs and rats, 359 about 75 million years ago (Adkins et al., 2001). 
360 The most dorsal deep retracting vibrissae muscle (PIP) submerges under the rows A and B

361 and is separated by a few hundred microns from the Pars maxillaris that runs toward rows $\mathrm{C}-$

362 E. Such separation may reflect compartmentalization of the guinea pig mystacial pad into the 363 nasal and maxillary parts that has not yet been described in guinea pigs.

364 Compartmentalization of the mystacial pad has been already observed in mice (Yamakado 365 and Yohro, 1979) and opossums (Grant et al., 2013), and it has been shown that nasal and 366 maxillary compartments of the mystacial pad develop from different growth centres in 367 embryo (Yamakado and Yohro, 1979).

368 In the guinea pig mystacial pad, the Partes media superior and inferior of the M. nasolabialis 369 profundus differ significantly from those in rats and mice. In rats and mice, these deep 370 protracting muscles are organized in to groups and can be observed between vibrissae rows 371 along the whole length of the mystacial pad (Haidarliu et al., 2010). They act on the more 372 caudal vibrissae especially, pulling them rostrally to reduce the spread of the whiskers overall 373 during protraction. In the guinea pig, a number of discrete bundles of muscle fibers can be 374 seen sliced transversally (in Fig. 7); however, these are only observed in the most rostral area 375 of the mystacial pad. We therefore conclude that guinea pigs do not have extrinsic protracting 376 muscles that would be analogous to those described in mice and rats.

\section{Behaviour}

378 The guinea pig moves its whiskers with a mean amplitude of $44 \pm 25.9$ degrees, which is 379 comparable to rats (43.19 \pm 7.65 degrees), but even larger than mice (31.25 \pm 11.64 degrees) 380 and opossums (36.04 \pm 9.53 degrees) (Mitchinson et al., 2011). The guinea pig positions its 381 whiskers with a mean offset angle of $98 \pm 12.5$ degrees, which is similar to the rat 382 (100.63 \pm 9.21 degrees) and opossum (94.42 \pm 9.01 degrees), but set slightly further back than 383 the mouse (112.53 \pm 6.85 degrees) (Mitchinson et al., 2011). While the range and position of 384 the whisker movements is fairly comparable to rats and mice, the movements themselves are 385 really rather different. The movements are rarely cyclic, and whisking is often absent, or only 386 occurs in short bouts of around three or four whisks and usually only unilaterally (Fig. 1B, 387 Fig. 9C), which agrees with previous observations of guinea pig whisker movements (Jin et 388 al., 2004). Indeed, guinea pig whisker movements are often asymmetric, occurring with head 389 rotations, and do not resemble the whisking motions observed in rats, mice and opossums.

390 The lack of whisking movements is probably associated with the thin and irregular intrinsic 
391 whisker muscles, causing the whiskers to move less often, compared to those of rats and 392 mice.

\section{Implications}

394 The total number of whiskers are reduced in the guinea pig (at 23 whiskers), which is a more 395 comparable amount to the marsupial opossum (23 whiskers), than to rats and mice (33

396 whiskers), despite them being closer related. Diurnal primates also have fewer whiskers (with 397 a minimum of 7 whiskers) that tend to be especially thin, with smaller whisker follicles

398 lacking in intrinsic muscles (Muchlinski 2010; Muchlinski et al., 2013), compared to 399 nocturnal primates (who have a minimum of 11 whiskers). In addition, the layout of the 400 whiskers tends to be disorganized in diurnal primates, who lack a clear grid-like arrangement 401 (Muchlinski et al., 2013). These aspects can also be observed in the guinea pig, but to a 402 slightly lesser extent, and might indicate common properties of a diurnal, visual lifestyle. 403 While there were no differences in the whisker follicle appearance, it was fairly large and 404 contained a sinus - the mystacial pad of the guinea pig was disorganized in terms of whisker 405 layout, intrinsic musculature and even innervation of the follicle. It might, therefore, be that 406 vibrissae organization, innervation distribution and whisker number are key predictors of 407 whisker specialisation in mammals, with whisker specialists, such as mice and rats, having 408 more whiskers that are better organized.

409 That the diurnal guinea pig still has large and sensitive whisker follicles, and can exert 410 movement over the whiskers using a complex architecture of intrinsic and extrinsic muscles, 411 indicates that the whiskers are functional in this animal, despite a greater reliance on vision.

412 Overall, the guinea pig mystacial pad is remarkably similar to rats and mice, despite them 413 moving their whiskers less and being ground-dwelling and diurnal. This might be due to 414 these animals being relatively closely related or, more likely, that the whiskers maintain an 415 important role for the guinea pig. Although being arboreal and nocturnal are important factors 416 in predicting the presence of intrinsic muscles, aspects of body size and other lifestyle 417 variables are also important influences (Mitchinson et al., 2011; Muchlinski et al., 2013), 418 such as being small and living in social groups (Muchlinski et al., 2013). Guinea pigs are 419 extremely social animals and live in large groups displaying quite complex social behaviours. 420 While whisker touch is implicated in social behaviours (Barnett, 2007; Muchlinski et al., 421 2013; Wolfe et al., 2011) this has not yet been explored in guinea pigs. It does seem likely 
422 that the whiskers could play an important role in aggressive and submissive interactions in

423 the guinea pig (for example, see figures in Grant and Mackintosh 1963).

424

425 Conclusions

426 In agreement with other studies on diurnal mammals, guinea pigs have fewer and less427 organized whiskers, than arboreal, nocturnal rodents. While the reduction in whisker number 428 and mystacial musculature suggests a larger reliance of the guinea pig on visual information, 429 overall, the mystacial pad is surprisingly similar to rat and mouse, indicating that the 430 whiskers may still play an important role in the life of the guinea pig. We suggest here that 431 protecting the eye and social touch behaviours are both roles that the whiskers might play in 432 guinea pig, and these will be important aspects of future research. Furthermore, we provide 433 evidence that vibrissae organization, in terms of mystacial musculature, follicle layout and 434 whisker number, is a key predictor of whisker specialisation in mammals.

436 Acknowledgements

437 The authors would like to thank undergraduate students Kayleigh Dean, Jennifer Lucas, 438 Gemma Parker, Alexander Parrish and Alasdair Glendinning for their help with the slicing 439 and staining. We are grateful for the technical help and guidance from Glenn Ferris, Dave 440 Maskew and Graham Tinsley. We are especially thankful to Eddie Gill and Heeley City 441 Farm, Sheffield for access to their animals for the behavioural filming, and Sam Watson for 442 the filming support.

\section{$444 \quad$ Literature Cited}

445 Adkins RM, Gelke EL, Rowe D and Honeycutt RL. 2001. Molecular phylogeny and 446 divergence time estimates for major rodent groups: evidence from multiple genes. Mol Biol 447 Evol 18:777-791.

448 Ahl AS. 1986. The role of vibrissae in behavior: a status review. Vet Res Commun 10: 245449268. 
450 Barnett SA. 2007. The rat: A study in behavior. Transaction Publishers.

451 Brecht M, Naumann R, Anjum F., Wolfe J, Munz M, Mende C, \& Roth-Alpermann C. 2011.

452 The neurobiology of Etruscan shrew active touch. Phil. Trans. R. Soc. B, 366:3026-3036.

453 Brown D, Wolfgang C. 2015. Tracker 4.8 xs. Cabrillo College.

454 http://www.cabrillo.edu/ dbrown/tracker/. Accessed 8th September 2015

455 Chatfield C. 2003. The analysis of time series: An introduction (6th ed.). London: Chapman 456 and Hall.

457 Dehnhardt G. 2002. Sensory systems. Marine mammal biology: An evolutionary approach. $458 \quad 116-41$.

459 Dehnhardt G, Hyvärinen H, Palviainen A, Klauer G. 1999. Structure and innervation of the 460 vibrissal follicle-sinus complex in the Australian water rat, Hydromys chrysogaster. J Comp 461 Neurol 41:550-62

462 Dörfl J. 1982. The musculature of the mystacial vibrissae of the white mouse. J Anat 463 135:147-154.

464 Grant RA, Haidarliu S, Kennerley NJ, Prescott TJ. 2013. The evolution of active vibrissal

465 sensing in mammals: evidence from vibrissal musculature and function in the marsupial

466 opossum Monodelphis domestica. J Exp Biol 216:3483-3494.

467 Grant RA, Arkley KP. 2016. Matched Filtering in Active Whisker Touch. In The Ecology of

468 Animal Senses. 59-82. Springer International Publishing.

469 Grant EC, Mackintosh JH. 1963. A comparison of the social postures of some common 470 laboratory rodents. Behaviour 21:246-259.

471 Graur D, Hide WA, Li WH. 1991. Is the guinea-pig a rodent? Nature 351:649 - 652.

472 Haidarliu S, Ahissar E. 1997. Spatial organization of facial vibrissae and cortical barrels in 473 the guinea pig and golden hamster. J Comp Neurol 385:515-527.

474 Haidarliu S, Simony E, Golomb D, Ahissar E. 2010. Muscle architecture in the mystacial pad 475 of the rat. Anat Rec 293:1192-1206. 
476 Haidarliu S, Kleinfeld D, Deschénes M, Ahissar E. 2015. The musculature that drives active 477 touch by vibrissae and nose in mice. Anat Rec 298:1347-1358.

478 Jin TE, Witzemann V, Brecht M. 2004. Fiber types of the intrinsic whisker muscle and 479 whisking behavior. J Neurosci 24:3386-3393.

480 Klingener D. 1964. The comparative myology of four dipodoid rodents (Genera Zapus, 481 Napeozapus, Sicista, and Jaculus). Misc Publ Mus Zool Univ Michigan 124:1-100.

482 Kulikov VF. 2011. A new vibrissa group in Insectivores (Mammalia, Insectivora) and its role 483 in orientation. Doklady Bio. Sci 438:154-157.

484 Luo ZX, Yuan CX, Meng QJ, Ji Q. 2011. A Jurassic eutherian mammal and divergence of 485 marsupials and placentals. Nature 476(7361):442-445.

486 Minkoff EC, Mikkelsen P, Cunningham WA, Taylor KW. 1979 The facial musculature of the 487 opossum (Didelphis virginiana). J Mammal 60:46-57.

488 Mitchinson B, Grant RA, Arkley KP, Perkon I, Prescott TJ. 2011. Active vibrissal sensing in 489 rodents and marsupials. Philosophical Transactions B. 366(1581):3037-3048.

490 Muchlinski MN, Durham EL, Smith TD, Burrows AM. 2013. Comparative histomorphology 491 of intrinsic vibrissa musculature among primates: implications for the evolution of sensory 492 ecology and “face touch". American journal of physical anthropology 150:301-312.

493 Muchlinski MN. 2010. A comparative analysis of vibrissa count and infraorbital foramen 494 area in primates and other mammals. Journal of Human Evolution. 58:447-73.

495 Muchlinski MN. 2008. The relationship between the infraorbital foramen, infraorbital nerve, 496 and maxillary mechanoreception: implications for interpreting the paleoecology of fossil 497 mammals based on infraorbital foramen size. Anat Rec 291:1221-1226.

498 Lyne A. 1959. The systematic and adaptive significance of the vibrissae in the Marsupialia. 499 Proc Zool Soc Lond 133:79-133.

500 Perkon I, Košir A, Itskov PM, Tasič J, Diamond ME. 2011. Unsupervised quantification of 501 whisking and head movement in freely moving rodents. J Neurophysiol 105:1950-1962.

502 Pocock RI. 1914. On the facial vibrissae of mammalia. Proc. Zool. Soc. Lond 84:889-912. 
503 Potter GE, Rabb EL, Jones WD, Hermann CL, Gibbs LW. 1957. The Muscular System of

504 Guinea Pig (Cavia porcellus). Bios:104-115.

505 Rice FL, Mance A, Munger BL. 1986. A comparative light microscopic analysis of the

506 sensory innervation of the mystacial pad. I. Innervation of vibrissal follicle-sinus complexes.

507 J Comp Neurol 252:154-174.

508 Rinker GC. 1954. The comparative myology of the mammalian genera Sigmodon,

509 Oryzomys, Neotoma, and Peromyscus (Cricetinae), with remarks on their intergeneric

510 relationships. Misc Publ Mus Zool Univ Michigan 83:1-25.

511 Sikich L, Woolsey TA, Johnson EM. 1986. Effect of a uniform partial denervation of the

512 periphery on the peripheral and central vibrissal system in guinea pigs. J Neurosci 6:1227-

5131240.

514 Welker WI. 1964. Analysis of sniffing of the albino rat. Behaviour 22:223-244.

515 Wineski LE. 1983. Movements of the cranial vibrissae in the golden hamster (Mesocricetus 516 auratus). J Zool 200: 261-280.

517 Wineski LE. 1985) Facial morphology and vibrissal movement in the golden hamster. J

518 Morph 183:199-217.

519 Wolfe J, Mende C, Brecht M. 2011. Social facial touch in rats. Behav Neurosci 125:900.

520 Woolsey TA, Welker C, Schwartz RH. 1975. Comparative anatomical studies of the SmL

521 face cortex with special reference to the occurrence of "barrels" in layer IV. J Comp Neurol $522 \quad 164: 79-94$.

523 Wong-Riley M. 1979. Changes in the visual system of monocularly sutured or enucleated 524 cats demonstrable with cytochrome oxidase histochemistry. Brain Res 171:11-28.

525 Yamakado M, Yohro T. 1979. Subdivision of mouse vibrissae on an embriological basis, 526 with descriptions of variations in the number and arrangement of sinus hairs and cortical

527 barrels in BALB/c (nu/p; nude, nu/nu) and hairless (hr/hr) strains. Am J Anat 155:153-174.

528 Yohro T. 1977. Arrangement and structure of sinus hair muscles in the big-clawed

529 shrew, Sorex unguiculatus. J Morphol 153:317-331.

530 
532 Figure 1. Recording and tracking guinea pig behaviour. A. The experimental set-up. The 533 high-velocity video camera above the arena, which was illuminated from below by an

534 infrared light box. B. An example of recording of whisker angles (nma: naïve mean angle) of 535 the left (in red) and right (in blue) whisker fields. Inset is the tracked video footage showing 536 head and whisker traces.

537 Figure 2. Layout of the mystacial vibrissae in a superficial tangential slice of the 538 mystacial pad of the guinea pig. Staining for cytochrome oxidase activity. (A1 - E5) 539 Follicles of the mystacial vibrissae $; \alpha-\delta$, straddler follicles; FBP, furry buccal pad; N, 540 nostril; N1 - N6, a row of follicles of the nasal (rhinal) vibrissae; POO, Pars orbicularis oris 541 of the M. buccinatorius; R, rostral; V, ventral. Scale bar $=1 \mathrm{~mm}$.

542 Figure 3. Intrinsic muscles in the rat (A) and guinea pig (B - E). A and $\mathbf{B}$ show the layout 543 of the mystacial pad of the rat and guinea pig, intrinsic muscles of the $\mathrm{C}$ row vibrissae are 544 indicated by black arrows, although intrinsic muscles are present throughout, from row A to 545 E in both rat and guinea pig. (N) Nasal compartment. (M) Maxilliary compartment. C. A 546 tangential slice of the mystacial pad showing intrinsic muscles at higher magnification, 547 including a straddling oblique intrinsic muscle (arrow head); D. enlarged boxed area in C; E. 548 row A and oblique intrinsic muscle between follicles of the vibrissae A1 and A2 (arrow 549 head). (1) Follicle sinus. Scale bars in $\mathbf{A}$ and $\mathbf{B}=1 \mathrm{~mm}, \mathbf{C}$ and $\mathbf{E}=0.5 \mathrm{~mm}$ and $\mathbf{D}=0.1 \mathrm{~mm}$. 550 All figure panels show tangential slices stained for cytochrome oxidase activity.

551 Fig. 4. Guinea pig whisker follicles of the C-row. A horizontal slice of the mystacial pad 552 stained with Masson's Trichrome. C1 - C4, whisker follicles; C, caudal, M, medial. (1) Ring 553 sinus; (2) ringwulst. Scale bar $=1 \mathrm{~mm}$.

554 Figure 5. Superficial vibrissa retracting extrinsic muscles of the guinea pig mystacial

555 pad. Tangential slices of the mystacial pad stained for cytochrome oxidase activity.

$556(\alpha, \beta, \gamma)$ Straddler follicles; B1, C1, vibrissal follicles; ML, M. maxillolabialis; NL, M.

557 nasolabialis; $\mathrm{R}$, rostral; $\mathrm{V}$, ventral. Scale bars $=0.5 \mathrm{~mm}$

558 Figure 6. Deep extrinsic vibrissa retracting muscles of the guinea pig. A tangential slice 559 of the mystacial pad stained for cytochrome oxidase activity. These muscles are part of the 560 M. nasolabialis profundus. A. A deep tangential slice of the mystacial pad. B. Enlarged boxed 561 area in (A). $(\alpha, \beta, \gamma, \delta)$ straddler follicles; (A1 - E2) vibrissa follicles. (1) Pars interna 
562 profunda; (2) Pars maxillaris; (3) Pars anterior; (4, 5) tapered ends of the muscle fibres of the

563 Pars interna profunda and Pars maxillaris, respectively, that are attached to the nasal

564 cartilage; $\mathrm{N}$, nostril; $\mathrm{R}$, rostral; $\mathrm{V}$, ventral. Scale bars $=1 \mathrm{~mm}$ in (A) and $0.5 \mathrm{~mm}$ in (B).

565 Figure 7. Deep extrinsic vibrissa protracting and retracting muscles of the guinea pig. A

566 tangential slice of the mystacial pad stained for cytochrome oxidase activity. A. A very deep

567 tangential slice of the mystacial pad. B and C. Enlarged boxed areas in A, respectively. D.

568 Collagen autofluorescence in the area shown in C. $(\alpha)$ straddler follicle; CF, collagenous

569 bundles of the deep fibrous mat; MB, muscle bundles; MF, muscle fibres; N, nostril; N1, a

570 follicle of the nasal vibrissae; PM, Pars maxillaris; PMI, pars media inferior; PMS, Pars

571 media superior; $\mathrm{R}$, rostral; $\mathrm{V}$, ventral. Scale bars $=1 \mathrm{~mm}$ in $(\mathbf{A}), 0.1 \mathrm{~mm}$ in $(\mathbf{B})$, and $0.5 \mathrm{~mm}$

572 in (C) 247 and (D)

573 Figure 8. Whisker movements in guinea pig. A. A histogram of whisker offset, the mean

574 angular position of the whiskers; B. a histogram of whisker amplitude, the amount the

575 whiskers move; C. an example trace of mean whisker angular positions from the left (in red)

576 and right (in blue) whisker fields.

577 
Figure 1. Recording and tracking guinea pig behaviour. A. The experimental set-up. The high-velocity video camera above the arena, which was illuminated from below by an infrared light box. B. An example of recording of whisker angles (nma: naïve mean angle) of the left (in red) and right (in blue) whisker fields. Inset is the tracked video footage showing head and whisker traces.

$$
\text { Figure } 1
$$

$79 \times 126 \mathrm{~mm}(150 \times 150 \mathrm{DPI})$ 


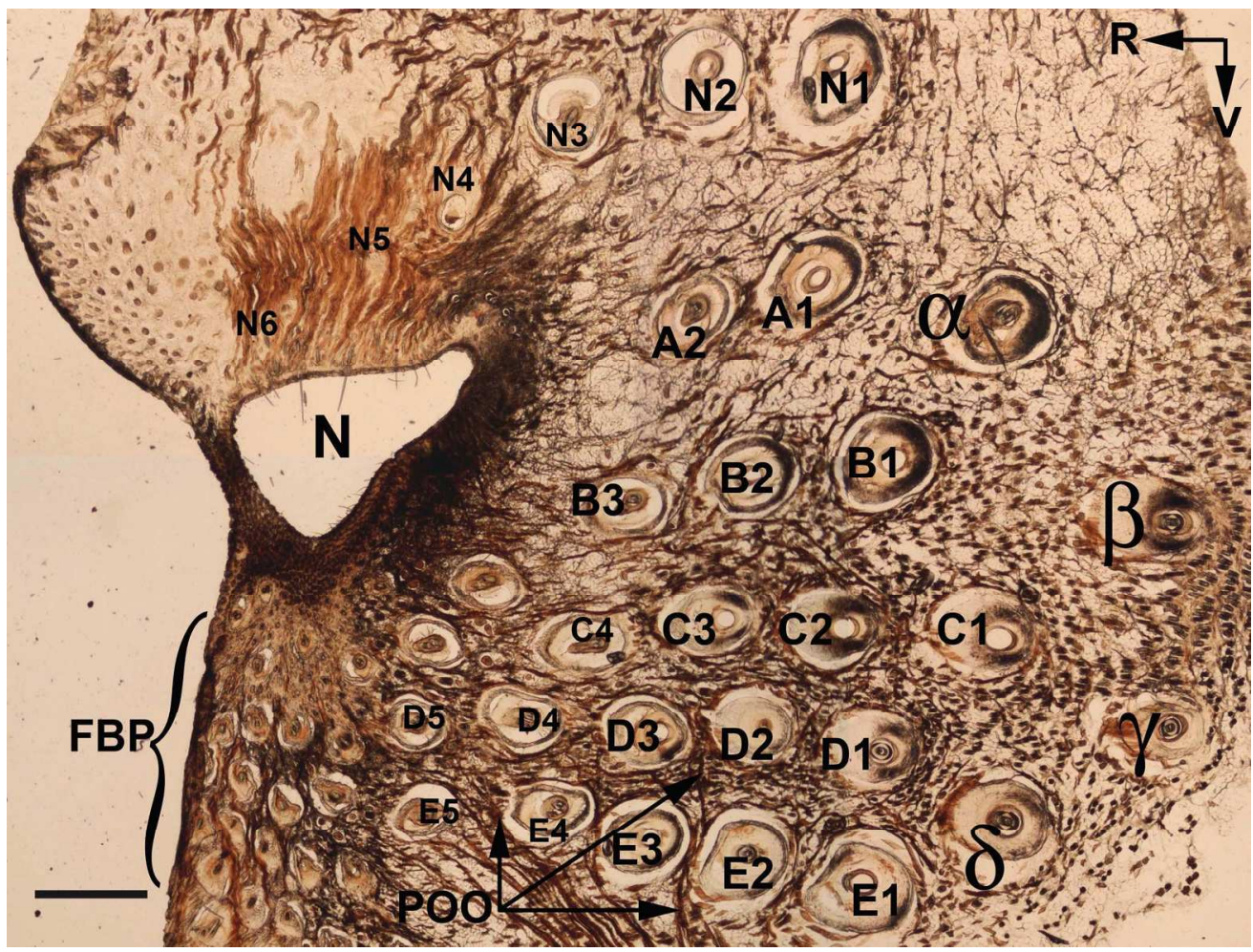

Figure 2. Layout of the mystacial vibrissae in a superficial tangential slice of the mystacial pad of the guinea pig. Staining for cytochrome oxidase activity. (A1 - E5) Follicles of the mystacial vibrissae; a- $\delta$ straddler follicles; FBP, furry buccal pad; N, nostril; N1 - N6, a row of follicles of the nasal (rhinal) vibrissae; POO, Pars orbicularis oris of the M. buccinatorius; $\mathrm{R}$, rostral; $\mathrm{V}$, ventral. Scale bar $=1 \mathrm{~mm}$. Figure 2 $180 \times 135 \mathrm{~mm}(300 \times 300 \mathrm{DPI})$ 

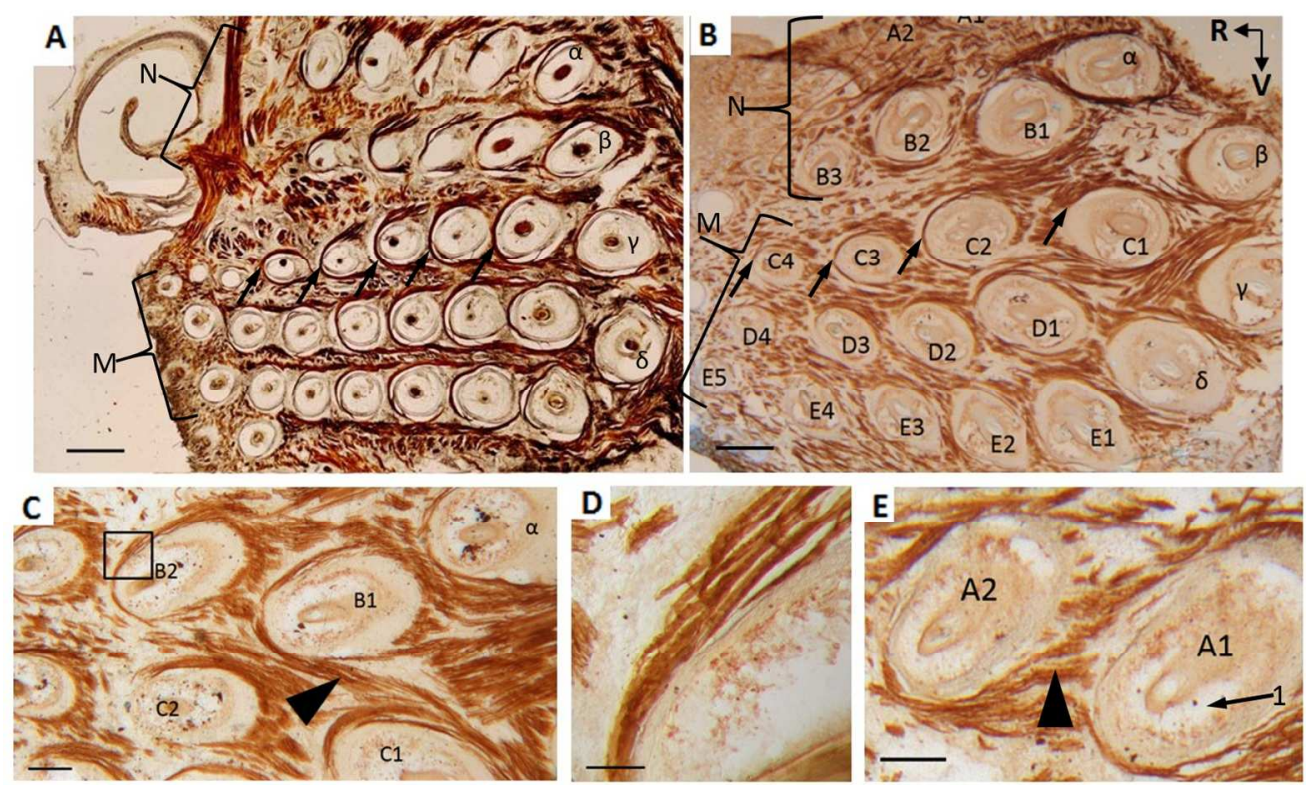

Figure 3. Intrinsic muscles in the rat $(A)$ and guinea pig $(B-E)$. A and $B$ show the layout of the mystacial pad of the rat and guinea pig, intrinsic muscles of the $C$ row vibrissae are indicated by black arrows, although intrinsic muscles are present throughout, from row $A$ to $E$ in both rat and guinea pig. (N) Nasal compartment. (M) Maxilliary compartment. C. A tangential slice of the mystacial pad showing intrinsic muscles at higher magnification, including a straddling oblique intrinsic muscle (arrow head); $D$. enlarged boxed area in C; E. row A and oblique intrinsic muscle between follicles of the vibrissae A1 and A2 (arrow head). (1) Follicle sinus. Scale bars in $A$ and $B=1 \mathrm{~mm}, C$ and $E=0.5 \mathrm{~mm}$ and $D=0.1 \mathrm{~mm}$. All figure panels show tangential slices stained for cytochrome oxidase activity

\section{Figure 3}

$272 \times 164 \mathrm{~mm}(150 \times 150 \mathrm{DPI})$ 


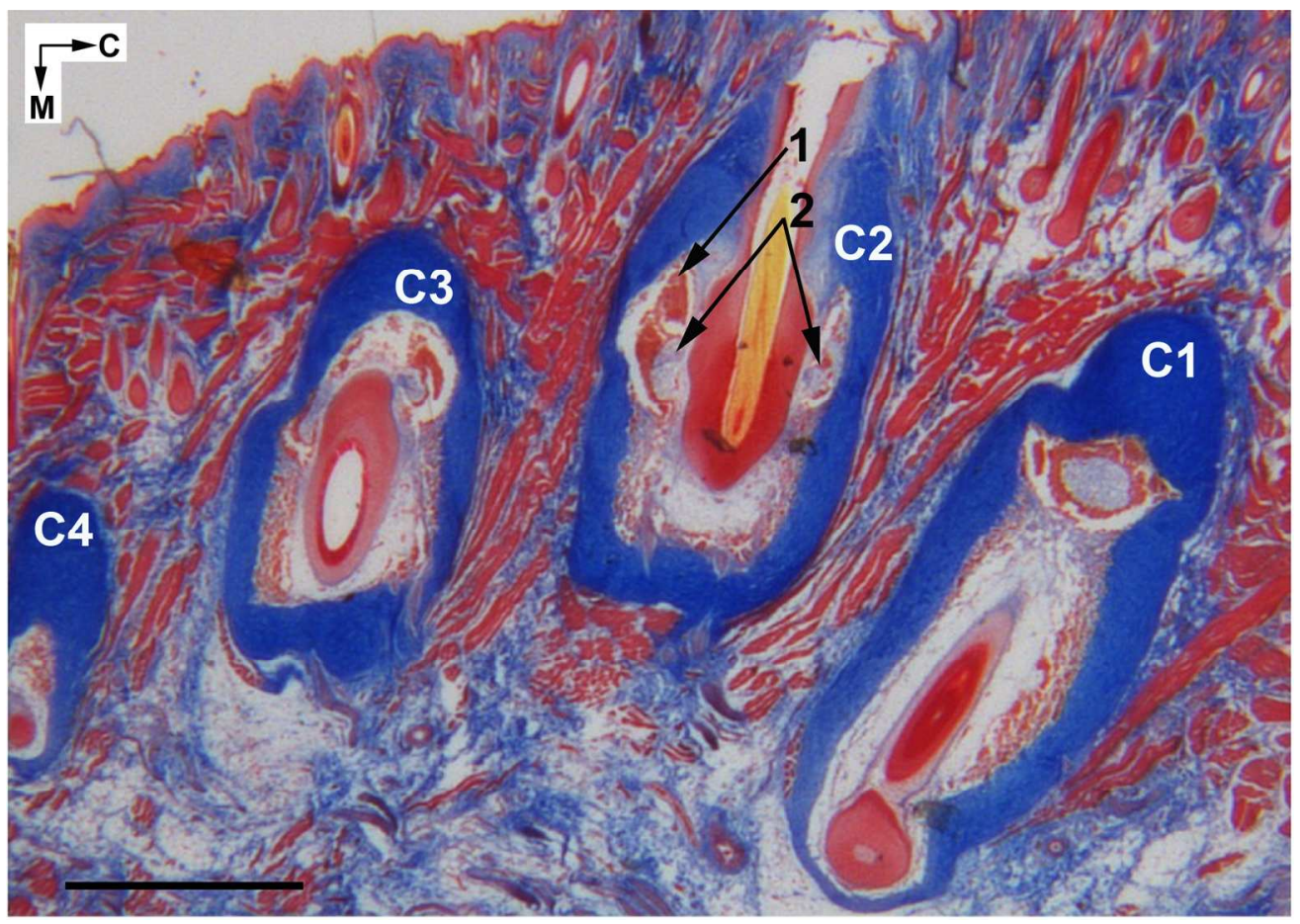

Fig. 4. Guinea pig whisker follicles of the C-row. A horizontal slice of the mystacial pad stained with Masson's Trichrome. C1 - C4, whisker follicles; C, caudal, M, medial. (1) Ring sinus; (2) ringwulst. Scale bar = $1 \mathrm{~mm}$. Figure 4 $170 \times 120 \mathrm{~mm}(300 \times 300 \mathrm{DPI})$ 

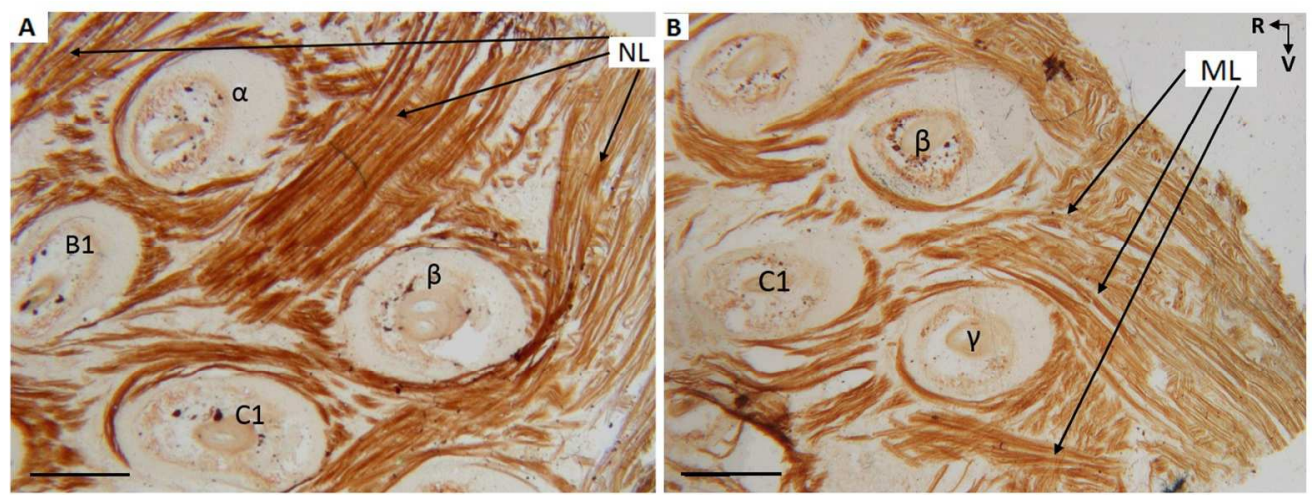

Figure 5. Superficial vibrissa retracting extrinsic muscles of the guinea pig mystacial pad. Tangential slices of the mystacial pad stained for cytochrome oxidase activity. ( $a, \beta, y)$ Straddler follicles; B1, C1, vibrissal follicles; ML, M. maxillolabialis; NL, M. nasolabialis; R, rostral; V, ventral. Scale bars $=0.5 \mathrm{~mm}$ Figure 5 $258 \times 98 \mathrm{~mm}(150 \times 150 \mathrm{DPI})$ 

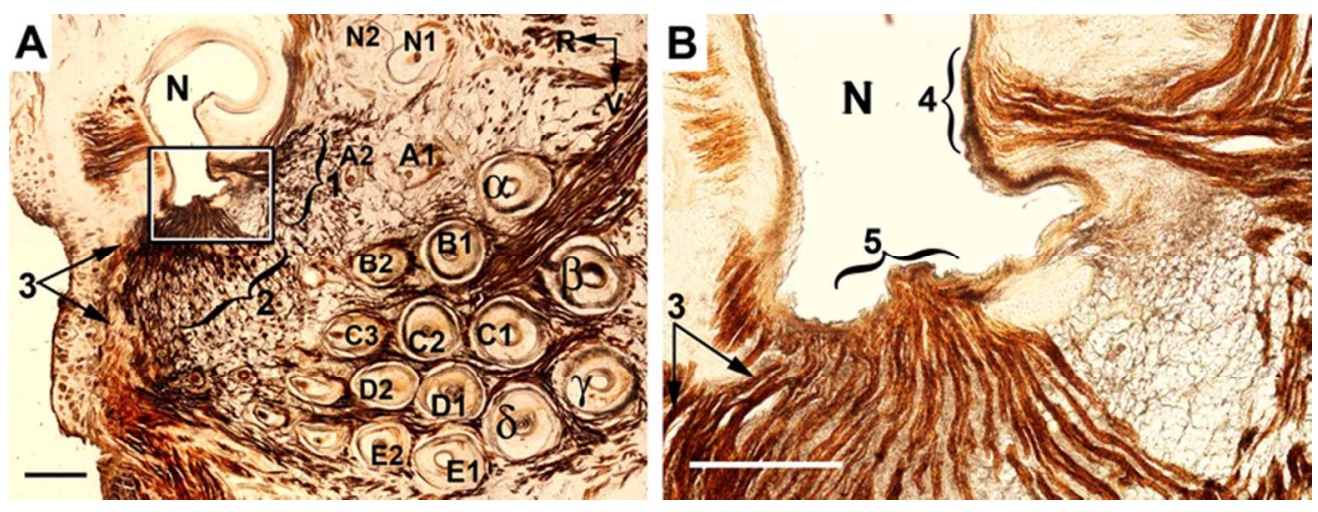

Figure 6. Deep extrinsic vibrissa retracting muscles of the guinea pig. A tangential slice of the mystacial pad stained for cytochrome oxidase activity. These muscles are part of the $M$. nasolabialis profundus. A. A deep tangential slice of the mystacial pad. B. Enlarged boxed area in (A). $(a, \beta, \gamma, \delta)$ straddler follicles; (A1 - E2) vibrissa follicles. (1) Pars interna profunda; (2) Pars maxillaris; (3) Pars anterior; $(4,5)$ tapered ends of the muscle fibres of the Pars interna profunda and Pars maxillaris, respectively, that are attached to the nasal cartilage; N, nostril; $R$, rostral; $V$, ventral. Scale bars $=1 \mathrm{~mm}$ in (A) and $0.5 \mathrm{~mm}$ in (B). Figure 6

$68 \times 25 \mathrm{~mm}(300 \times 300 \mathrm{DPI})$ 

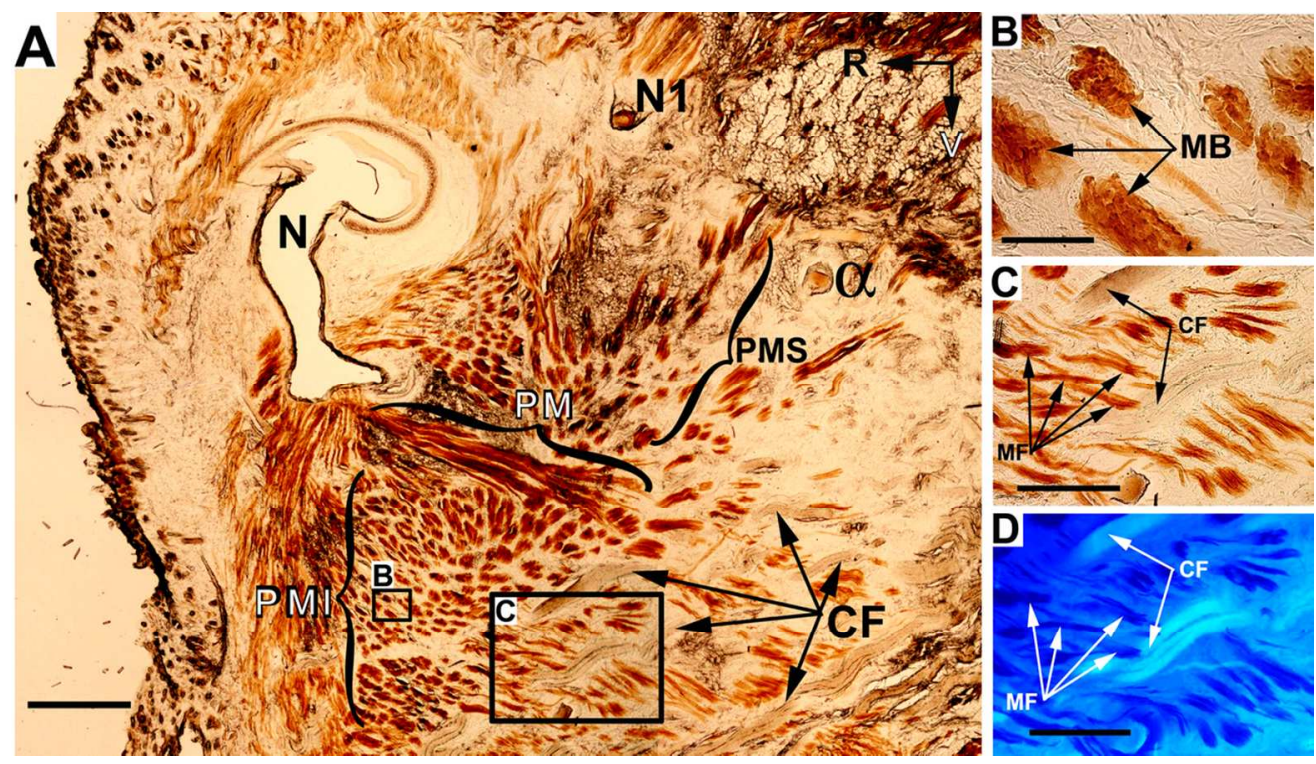

Figure 7. Deep extrinsic vibrissa protracting and retracting muscles of the guinea pig. A tangential slice of the mystacial pad stained for cytochrome oxidase activity. A. A very deep tangential slice of the mystacial pad. B and C. Enlarged boxed areas in A, respectively. D. Collagen autofluorescence in the area shown in $\mathrm{C}$.

(a) straddler follicle; $C F$, collagenous bundles of the deep fibrous mat; $M B$, muscle bundles; MF, muscle fibres; N, nostril; N1, a follicle of the nasal vibrissae; PM, Pars maxillaris; PMI, pars media inferior; PMS, Pars media superior; $R$, rostral; V, ventral. Scale bars = $1 \mathrm{~mm}$ in (A), $0.1 \mathrm{~mm}$ in (B), and $0.5 \mathrm{~mm}$ in (C) 247 and (D)

Figure 7

$108 \times 61 \mathrm{~mm}(300 \times 300 \mathrm{DPI})$ 


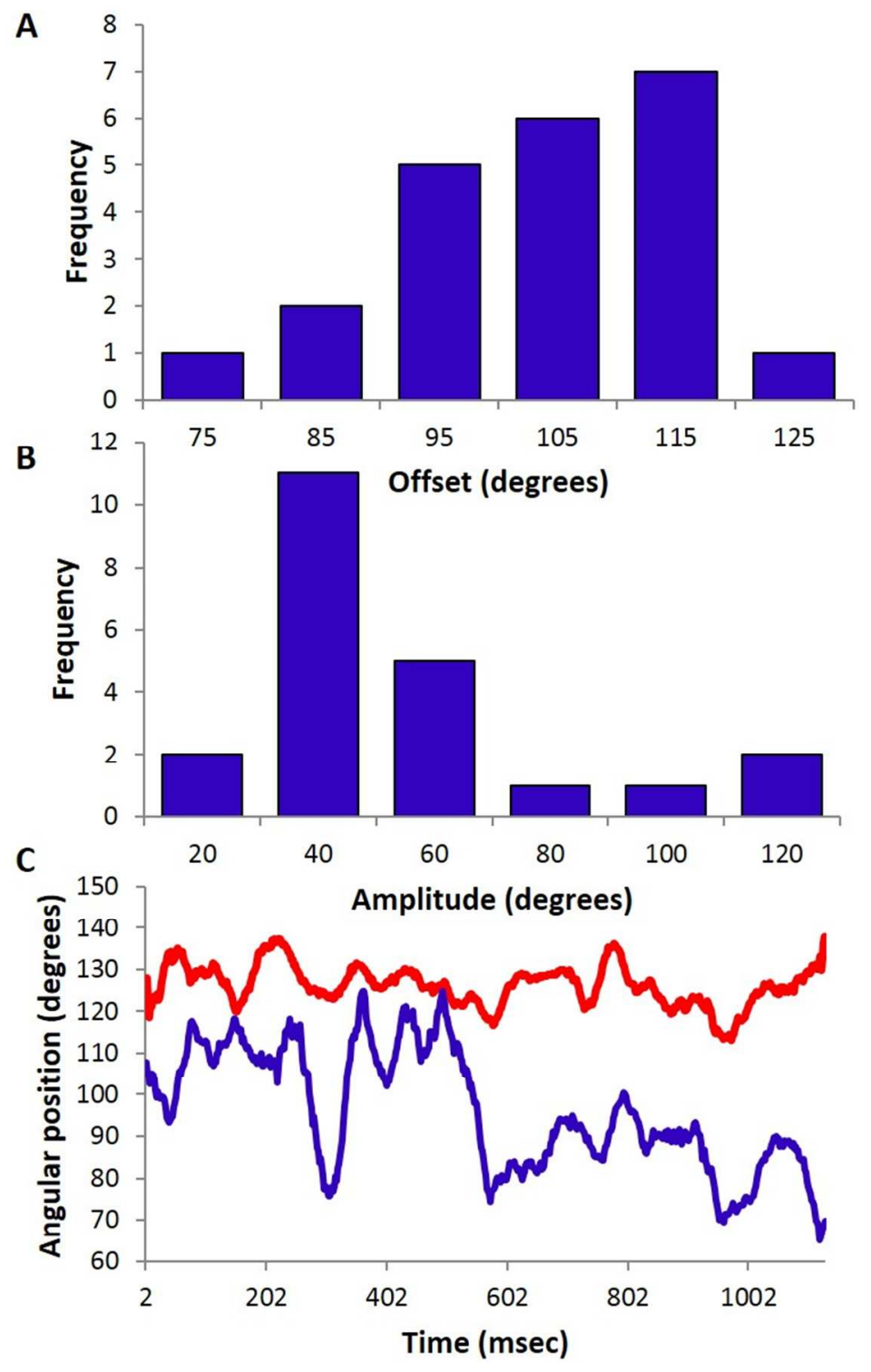

Figure 8. Whisker movements in guinea pig. A. A histogram of whisker offset, the mean angular position of the whiskers; B. a histogram of whisker amplitude, the amount the whiskers move; $\mathrm{C}$. an example trace of mean whisker angular positions from the left (in red) and right (in blue) whisker fields.

Figure 8

$131 \times 210 \mathrm{~mm}(150 \times 150 \mathrm{DPI})$

John Wiley \& Sons, Inc. 